\title{
Exercise-induced bronchial hyperresponsiveness and parental ISAAC questionnaire responses
}

\author{
A-L. Ponsonby*, D. Couper*, T. Dwyer*, A. Carmichael**, R. Wood-Baker+
}

Exercise-induced bronchial hyperresponsiveness and parental ISAAC questionnaire responses. A-L. Ponsonby, D. Couper, T. Dwyer, A. Carmichael, R. Wood-Baker. (CERS Journals Ltd 1996.

ABSTRACT: The predictive value of parental questionnaire responses for exerciseinduced bronchoconstriction in childhod asthma has not been fully clarified. The aim of this study was to compare exercise-induced bronchial hyperresponsiveness in 7 year old children with parental responses to core questions in the International Study of Asthma and Allergies in Childhood (ISAAC) study.

A cross-sectional study was conducted on 191 (91\% of eligible) children from seven randomly selected schools in Southern Tasmania. Study measurements included a parental questionnaire and exercise challenge testing, using a recently validated 6 min free-running protocol. The response to exercise was assessed using forced expiratory volume in one second (FEV1) measurement.

The median percentage fall in FEV1 was significantly higher in children whose parents responded positively to ISAAC questions on a history of wheeze $(p=0.0031)$ or asthma $(p=0.0005)$, recent wheeze $(p=0.0005)$, sleep disturbance due to wheeze $(p=0.0005)$, or exercise-induced wheeze $(p=0.0015)$. Receiver operating characteristic (ROC) curve analysis showed exercise-induced bronchial hyperresponsiveness to be a good indicator of current asthma status. Using a $12 \%$ or greater fall in FEV1 postexercise as a positive test response, the exercise challenge had sensitivity and specificity estimates for current asthma and exercise-induced wheeze of $(0.58$ and $0.77)$ and $(0.60$ and 0.77$)$, respectively.

In conclusion, the respiratory response to exercise was consistent with parental responses to the ISAAC questionnaire in a population-based sample of 7 year old children. These findings will assist interpretation of large ISAAC studies in terms of asthma prevalence.

Eur Respir J., 1996, 9, 1356-1362.
*Menzies Centre for Population Health Research, University of Tasmania, Hobart, Tasmania, Australia. **Depts of Paediatrics and Child Health, and + Medicine, University of Tasmania, Hobart, Tasmania, Australia.

Correspondence: A-L. Ponsonby

Menzies Centre for Population Health Research

University of Tasmania

17 Liverpool Street

Hobart

Tasmania

Australia 7000

Keywords: Childhood asthma exercise

International Study of Asthma and Analysis and Allergies in Childhood

receiver operating characteristic analysis

Received: October 121995

Accepted after revision April 11996

This project was funded by the Royal Hobart Hospital Research Fund.
Asthma is one of the most important diseases in childhood. There is now considerable concern that the prevalence of asthma and allergic diseases is increasing in Western and developing countries [1,2]. The International Study of Asthma and Allergies in Childhood (ISAAC) has been developed to provide a standardized approach to international and regional comparisons of asthma prevalence and severity, and has been described in detail previously $[1,3]$. The ISAAC design comprises three phases. Phase 1 uses core questionnaires designed to assess the prevalence and severity of asthma and allergic disease in defined populations [1]. The ISAAC written questionnaire contains a core module of eight questions on asthma and wheeze for 7 year olds. The module is based on questions drawn from the International Union Against Tuberculosis and Lung Disease (IUATLD) questionnaire [4], and from other surveys [5-7]. The first formal validation of the ISAAC written questionnaire has recently been published [8]. Sensitivity and specificity estimates for self-response to ISAAC questions in relation to bronchial hyperresponsiveness (BHR) to methacholine were determined on 87 children aged $13-15$ yrs. Overall, the ISAAC and IUATLD written questionnaires had similar effectiveness in predicting BHR in the adolescent age group [8]. Data obtained from using the ISAAC questionnaire in different settings and age-groups against different biological measures of asthma will be important for the validation and evaluation of the ISAAC study results.

Exercise is one of the most common precipitants of asthma, particularly in children because of their high levels of physical activity [9]. The pathogenesis of exercise-induced asthma is thought to be closely associated with the fluxes in heat and water that develop within the tracheobronchial tree during the warming and humidification of large volumes of air [10, 11]. HABY et al. [12] have developed an exercise challenge protocol suitable for epidemiological studies of asthma in children. They found BHR following exercise challenge (a fall in forced expiratory volume in one second (FEV1) of $\geq 13 \%$ following 6 min running) was in good agreement with BHR following histamine challenge (a fall in FEV $1 \geq 20 \%$ ). Agreement between the two tests was $0.87(0.80-0.94)$ [12). For a positive parental response to a question on recent wheeze, both tests had a sensitivity of 0.27 and a specificity of 0.94 [12]. HABY et al. [12] stated that 
the cut-off point they used (a 13\% fall in FEV1 following exercise) required confirmation as the most sensitive and specific point. The receiver operating characteristic (ROC) curve is a useful way to express the relationship between sensitivity and specificity for a given test and can assist the decision in determining an optimal cut-off point for a test to discriminate between diseased and nondiseased individuals [13]. The aim of this study was to compare the results of a physiological test of bronchial responsiveness by exercise testing with parental responses to the ISAAC questionnaire in a sample of 7 year old children using ROC analysis.

\section{Methods}

Tasmania is the southern island state of Australia, with a cool temperate climate. A cross-sectional study was performed in the last school term from September to December 1994. A sample of children was selected from seven randomly selected primary schools (Government and privately funded) in Southern Tasmania. Questionnaires were distributed through the schools to parents of all 7 year old children in each selected school. If the parents consented to their child's participation in the exercise test, then the exercise test was performed after completion of the parental questionnaire. The study was approved by the Human Ethics Committee of the University of Tasmania.

\section{Sample}

Two hundred and nine children were eligible for inclusion, being 7 years of age at study commencement in a selected school. These eligible children and their families were invited to participate in the project. Ten children and their families did not wish to participate. For four children, the parental questionnaire was completed but consent was not obtained for exercise testing. The remaining 195 children and their families completed the questionnaire and consented to exercise challenge participation. Four of these children did not perform the exercise testing for the following reasons: low baseline FEV1; physical disability (wheelchair bound); and lack of co-operation (two children). Thus, exercise challenge data were available on 191 (91\%) of the eligible children. Parental questionnaire data were available on 199 $(95 \%)$ of the eligible children, but not all questions were completed by all parents. The sample consisted of 95 boys and 104 girls, of whom 185 (93\%) were Caucasian. The mean interval between date of completion of the questionnaire and exercise testing was 6.9 days (SD 12.1).

\section{The parental questionnaire}

The questionnaire included information on the following variables in sequence: identification; questions from the 1968 Tasmanian Asthma Survey on 7 year olds [14] (history of eczema, food or medicine allergy, hives, asthma or wheezy breathing and hay fever); the eight core questions on asthma and wheeze from the ISAAC protocol (table 1); asthma medication use; home environment; diet; and parental history of asthma or wheezing or hay fever.

\section{Respiratory measurements}

Indoor and outdoor ambient temperature and relative humidity were recorded at hourly intervals during exercise testing. Lung function was measured with two compact Alpha spirometers (Vitalograph Ltd, UK). The spirometers were calibrated at the start of each session of exercise testing and checked hourly to ensure the machine measured the calibration volume to within 3\%. Height and weight were measured prior to baseline lung function.

The children were shown a video, which demonstrated the respiratory manoeuvre necessary for measurement of forced expiratory volume. All lung function tests were performed with the child standing and without a noseclip. Children were instructed to take a maximal breath in and then blow out as hard and as fast as they could. After further instruction and two practice attempts, each child performed three forced expiratory manoeuvres [15]. Forced expiratory manoeuvres were repeated until two measurements of FEV1 within $100 \mathrm{~mL}$ of each other were obtained. For children receiving beta-agonists or other asthma medication, these were withheld for $6 \mathrm{~h}$ prior to the exercise challenge test. For each child, the baseline FEV1 was compared with that predicted for height and sex using an Australian nomogram [12]. If the observed FEV1 was less than $75 \%$ of the predicted value and also below three standard deviations from the expected value for the child's height and sex, then the child was excluded from the test. The second criterion was included because percentage predicted FEV1 alone does not take into account the distribution of values at each height in the reference population [16].

\section{The exercise challenge}

The exercise protocol developed by HABY et al. [12] was used as a basis. Briefly, the children ran for $6 \mathrm{~min}$ on the $100 \mathrm{~m}$ track of a flat, grass circle marked with cones $10 \mathrm{~m}$ apart. Each child wore a noseclip to ensure mouth-breathing and a heart rate monitor (Polar Electro, Finland) for the duration of the run. Heart rate was recorded at intervals of $1 \mathrm{~min}$ or less. The children were instructed to reach and then maintain a heart rate of at least 180 beats $\cdot \mathrm{min}^{-1}$. The heart rate monitors were set to emit an auditory signal while the heart rate was above 179 beats $\cdot \min ^{-1}$. The distance run by each child was measured to provide, in conjunction with body weight, an indirect estimation of oxygen consumption [17].

Following exercise, FEV1 measurements were made at 3,5 and $10 \mathrm{~min}$. These time-points were chosen to encompass the variable bronchoconstrictor response to exercise [18]. At each point, three expiratory manoeuvres were performed. If the child felt unwell or distressed during the run or lung function testing, then the child was examined by a doctor, rested and given $200 \mu \mathrm{g}$ of salbutamol aerosol if required. The child was then reassessed $10 \mathrm{~min}$ after bronchodilator therapy.

\section{Statistical analysis}

The study sample was obtained by cluster sampling. Seven schools were selected at random from primary schools in the Southern Region of the state. Questionnaires 
were sent to the parents of all 7 year old children at the selected schools. The response to exercise was defined as the postexercise FEV1, expressed as a percentage of the baseline FEV1. The baseline FEV1 was defined as the highest pre-exercise measurement [19]. The number of readings taken varied across children. At least three readings were taken, with more being taken if the largest two were not within $100 \mathrm{~mL}$ of one another or if the largest one fell below the child's practice attempts. At each of the 3, 5 and 10 min points postexercise, three FEV1 readings were taken, with the maximum of the three regarded as the FEV1 at that time. The minimum of these three maxima was taken as the postexercise FEV1. As with any experimental procedure, there is some natural variation inherent in the measurement of lung function. Consequently, using three postexercise times (and the fact that the maximum reading was taken from three or more readings for the baseline FEV1) means that there is likely to be an apparent fall in FEV1 even if there is no real change in lung function. This should be borne in mind when looking at the percentage fall values reported in this paper. However, this procedure does not introduce systematic bias between groups compared in the analyses, so results for differences between groups remain valid.

The distribution of the percentage fall in FEV1 was markedly skewed, with a long upper tail. A few children experienced a very large percentage fall in FEV1. To reduce the effects of these extreme values, medians rather than means were estimated, and nonparametric tests were used when comparing percentage fall in FEV1 between groups. The Wilcoxon test [20] was used when grouping was done using dichotomous variables, while the Kruskal-Wallis test [20] was used for those grouping variables with more than two categories. Multiple linear regression models [20] were used to examine the effect of the temperature, humidity and other variables on baseline FEV1 and response to exercise. Residuals from these models were plotted to investigate the possibility of nonlinear relationships.

ROC curves [13, 20] were developed to allow a visual assessment of the relationship between exercise response and parental response to asthma questions over a range of cut-off points for classification of exercise-induced bronchial hyperresponsiveness. The sensitivity and specificity were calculated for a range of cut-off points. The $\mathrm{x}$-axis, "1-Specificity", is the false positive rate, and the y-axis, "Sensitivity", is the true positive rate. The cut-off points closest to the upper left hand corner of the graphs were determined using a compass to determine the shortest line which intersected the left hand corner of the graph and the ROC curve. The overall accuracy of the exercise challenge in relation to parental questionnaire response is reflected by the area under each curve. Summary measures of the validity of exercise response in relation to different questions were estimated using Youden's Index [21], which is calculated as the sum of sensitivity and specificity minus one.

\section{Results}

\section{Exercise challenge}

The mean distance completed was 851 (SD 108) m. One hundred and ninety one children had a satisfactory heart rate of at least $180 \pm 10$ beats $\cdot \mathrm{min}^{-1}$ during the last $4 \mathrm{~min}$ of exercise. One child had an average rate of only 169 beats $\cdot \mathrm{min}^{-1}$ for the last $4 \mathrm{~min}$ but ran $940 \mathrm{~m}$. This child was included in the analyses. Three children developed asthma symptoms and had to stop the test after completing 450, 400 and $300 \mathrm{~m}$. These children responded to exercise with a fall in FEV1 of 59, 39 and 39\%, respectively, measured $3 \mathrm{~min}$ after ceasing to run. They were included in the FEV1 analyses. The children ran in varying conditions, with median outdoor temperature 14 (range 9$27)^{\circ} \mathrm{C}$ and median outdoor humidity $70(32-100) \%$. The median indoor temperature and humidity were $16(14-26)^{\circ} \mathrm{C}$ and $58(32-80) \%$. The indoor temperature was usually higher than outdoor temperature (median difference 3 $(-3-8)^{\circ} \mathrm{C}$ ) and the indoor humidity was usually lower than outdoor humidity (median difference -14 (-44-18) $\%)$. Using linear regression, neither indoor nor outdoor temperature or humidity predicted exercise response. When all four terms were considered together, they explained less than $2 \%$ of the variance in percentage fall in FEV1. In addition, temperature and humidity were not related to baseline FEV1.

The fall in FEV1 was not normally distributed, with a longer upper tail than lower tail. The median fall in FEV1, postexercise, was 7.7 (range -11 to 61 )\%. As discussed previously (see Statistical methods), part of this apparent fall is due to the method of comparison of the lowest of the three maximal FEV1 recorded postexercise to the maximal preexercise FEV1. For children with no history of asthma, recent wheeze or any past history of asthma or wheezy breathing $(n=103)$, the percentage fall in FEV1 was approximately normally distributed and the upper limit of the $95 \%$ confidence interval for this normal group (mean \% fall $+1.96 \times \mathrm{SD}$ ) was $21 \%$.

Table 1. - Distribution of responses to ISAAC questions for wheezing and asthma

\begin{tabular}{|c|c|}
\hline $\begin{array}{l}\text { Question } \\
\text { (Response for which prevalence is calculated) }\end{array}$ & $\begin{array}{l}\text { Prevalence } \\
\text { of response }\end{array}$ \\
\hline $\begin{array}{l}\text { 1. Has your child ever had wheezing or } \\
\text { whistling in the chest at any time in the } \\
\text { past? (Yes) }\end{array}$ & $\begin{array}{c}42 \% \\
83 / 197\end{array}$ \\
\hline $\begin{array}{l}\text { 2. Has your child had wheezing or whistling } \\
\text { in the chest in the last } 12 \text { months (Yes) }\end{array}$ & $\begin{array}{c}21 \% \\
41 / 199\end{array}$ \\
\hline $\begin{array}{l}\text { 3. How many attacks of wheezing has your child } \\
\text { had in the last } 12 \text { months? (None) }\end{array}$ & $\begin{array}{c}81 \% \\
160 / 197\end{array}$ \\
\hline $\begin{array}{l}\text { 4. In the last } 12 \text { months, how often, on average, } \\
\text { has your child's sleep been disturbed due to } \\
\text { wheezing? (Never) }\end{array}$ & $85 \%$ \\
\hline (Less than one night per week) & $\begin{array}{l}169 / 198 \\
11 \% \\
22 / 198\end{array}$ \\
\hline (One or more nights per week) & $\begin{array}{l}3.5 \% \\
7 / 198\end{array}$ \\
\hline $\begin{array}{l}\text { 5. In the last } 12 \text { months, has wheezing ever been } \\
\text { severe enough to limit your child's speech to one } \\
\text { or two words at a time between breaths? (Yes) }\end{array}$ & $\begin{array}{c}3 \% \\
6 / 199\end{array}$ \\
\hline 6. Has your child ever had asthma? (Yes) & $\begin{array}{c}29 \% \\
58 / 199\end{array}$ \\
\hline $\begin{array}{l}\text { 7. In the last } 12 \text { months, has your child's chest } \\
\text { sounded wheezy during or after exercise? (Yes) }\end{array}$ & $\begin{array}{c}13 \% \\
26 / 196\end{array}$ \\
\hline $\begin{array}{l}\text { 8. In the last } 12 \text { months, has your child had a dry } \\
\text { cough at night, apart from a cough associated with a } \\
\text { cold or chest infection? (Yes) }\end{array}$ & $\begin{array}{c}24 \% \\
48 / 197\end{array}$ \\
\hline
\end{tabular}


The contribution of body weight, velocity and distance run to percentage fall in FEV1 was examined. The three children who stopped running early because of asthma symptoms were excluded. Velocity $(\mathrm{p}=0.7870)$ and distance run $(p=0.7191)$ were not significantly related to percentage fall in FEV1. After adjustment for baseline FEV1 and distance run, height was inversely related to percentage fall in FEV1 $(p=0.046)$. Weight was also inversely related to percentage fall in FEV1 $(\mathrm{p}=0.017)$ after adjustment for baseline FEV1 and distance run. The inverse relationship between weight and exercise response and height and exercise response was stronger for the group of children with current asthma than children without current asthma ( $\mathrm{p}=0.031$ and $\mathrm{p}=0.067$, respectively). The lowest of the minimum FEV1 readings was recorded for the reading set $3 \mathrm{~min}$ after exercise. For children with current asthma, median minimum FEV1 values at baseline and 3, 5 and $10 \mathrm{~min}$ postexercise were $1.49,1.29,1.31$ and 1.35 $\mathrm{L}$, respectively. For children without current asthma, the corresponding values were $1.48,1.39,1.42$ and $1.42 \mathrm{~L}$.

\section{Prevalence of asthma and respiratory symptoms by ques- tionnaire}

The prevalence of asthma and other respiratory symptoms are shown in table 1 . With regard to other features of atopy, $22 \%$ (44 out of 197) had infantile eczema and $27 \%$ (53 out of 198) had had eczema in the creases of elbows, wrists or knees. Twenty percent (39 out of 195) were reported to get attacks of hay fever, and 36\% (71 out of 199) had suffered from asthma or wheezy breathing.

\section{Relationship between exercise and questionnaire responses}

Table 2 compares the median change in FEV 1 for those with a positive history of symptoms to the group of children with a negative history. The median percentage fall in FEV1 was examined for questions from ISAAC (table 2 ). The percentage fall in FEV1 varied by the frequency of use of asthma medication. For those children who did not use asthma medication in the preceding 12 months $(n=156)$, the median fall in FEV1 was $8.6 \%$; for those using asthma medication less than once a month $(n=21)$, $17.2 \%$; for those using asthma medication more than once a month, $17.2 \%$; and for those children on daily asthma medication $(n=5)$, the median fall in FEV1 was $33.2 \%$. The median fall in FEV1 did not differ by school $(\mathrm{p}=0.9064)$, gender $(\mathrm{p}=0.5240)$, the child's report of a cold $(\mathrm{p}=0.6503)$, the observer's report of signs of respiratory illness $(\mathrm{p}=$ 0.9365 ), whether the child had exercised vigorously in the $4 \mathrm{~h}$ preceding the test $(\mathrm{p}=0.1744)$, or used any medication within $6 \mathrm{~h}$ of the test $(\mathrm{p}=0.8748)$. No children used asthma medication in the $6 \mathrm{~h}$ prior to the test.

ROC curves were constructed to examine the performance of the exercise challenge test to ISAAC questionnaire responses. ROC curves were used because they provide information on the sensitivity and specificity of the exercise test using various cut-off points for exercise-induced BHR in terms of the percentage fall in FEV1 postexercise. Figure 1 displays ROC curves for the exercise test in relation to recent wheeze (ISAAC Question 2) and exercise wheeze (ISAAC Question 7). For exercise wheeze, the cut-off points closest to the upper left hand corner of the graph (1.0 sensitivity, 1.0 specificity) correspond to an FEV1 fall postexercise of 11 or $12 \%$. Using a $12 \%$ fall in FEV1 to classify exercise-induced BHR, the test had a sensitivity of 0.54 and a specificity of 0.78 for the history of recent wheeze or whistle in the chest. The ROC curve for exercise-related wheeze (ISAAC Question 7) was horizontal for FEV1 falls of 7-3\%, indicating an increase in the false positive rate with no improvement in sensitivity. Figure 2 displays ROC curves for the exercise test in relation to history of asthma

Table 2. - Relationship between the respiratory response to exercise and questionnaire history

\begin{tabular}{|c|c|c|c|c|c|}
\hline \multirow[b]{2}{*}{ Factor } & \multicolumn{2}{|c|}{ Factor present } & \multicolumn{2}{|c|}{ Factor absent } & \multirow[b]{2}{*}{ p-value } \\
\hline & $\mathrm{n}$ & $\begin{array}{c}\text { Median } \\
\% \text { fall } \\
\text { FEV1 }\end{array}$ & $\mathrm{n}$ & $\begin{array}{c}\text { Median } \\
\% \text { fall } \\
\text { FEV1 }\end{array}$ & \\
\hline $\begin{array}{l}\text { History of eczema in the creases of } \\
\text { elbows, wrists or knees }\end{array}$ & 51 & 9.8 & 139 & 7.1 & 0.0132 \\
\hline History of asthma or wheezy breathing & 68 & 11.2 & 123 & 6.3 & 0.0001 \\
\hline History of hay fever & 38 & 10.2 & 152 & 7.4 & 0.0599 \\
\hline $\begin{array}{l}\text { History of ever having wheeze or } \\
\text { whistle in chest (ISAAC Q1) }\end{array}$ & 80 & 9.6 & 109 & 6.6 & 0.0031 \\
\hline $\begin{array}{l}\text { History of recent* wheeze or whistle in } \\
\text { chest (ISAAC Q2) }\end{array}$ & 39 & 12.3 & 152 & 7.0 & 0.0005 \\
\hline $\begin{array}{l}\text { History of sleep disturbance due to } \\
\text { wheeze (ISAAC Q4) }\end{array}$ & 28 & 15.6 & 162 & 7.1 & 0.0005 \\
\hline $\begin{array}{l}\text { History of recent* speech limitation } \\
\text { because of wheeze (ISAAC Q5) }\end{array}$ & 5 & 12.7 & 186 & 7.6 & 0.0566 \\
\hline History of ever having asthma (ISAAC Q6) & 56 & 11.2 & 135 & 6.7 & 0.0005 \\
\hline $\begin{array}{l}\text { History of recent wheeze during or after } \\
\text { exercise (ISAAC Q7) }\end{array}$ & 25 & 12.4 & 163 & 6.9 & 0.0015 \\
\hline $\begin{array}{l}\text { History of recent dry cough at night, apart } \\
\text { from a cold or chest infection (ISAAC Q8) }\end{array}$ & 46 & 10.6 & 143 & 7.1 & 0.0617 \\
\hline Mother has smoked since child's birth & 55 & 9.4 & 127 & 11.0 & $0.6240^{+}$ \\
\hline Father has smoked since child's birth & 58 & 11.4 & 120 & 9.9 & $0.0811^{+}$ \\
\hline
\end{tabular}

*: recent refers to the previous 12 months; +: Kruskal-Wallis test used as missing responses were treated as a separate category.

ISAAC: International Study of Asthma and Allergies in Childhood; FEV1: forced expiratory volume in one second. 


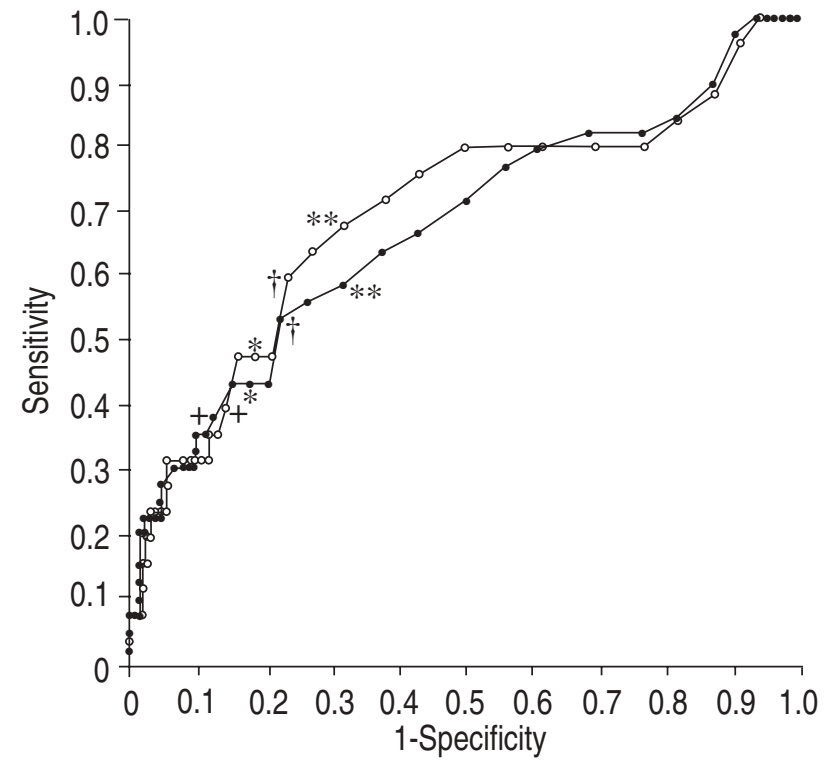

Fig. 1. - A comparison of the exercise challenge test with wheeze history measured by parental questionnaire. - - - child's chest has sounded wheezy during or after exercise in the past 12 months (Q7); - - : child has had wheezing or whistling in the chest in the last 12 months (Q2); fall in FEV1 post exercise ${ }^{+}:-16 \%$; *: $-14 \%$; $-12 \%$; **: $-10 \%$.

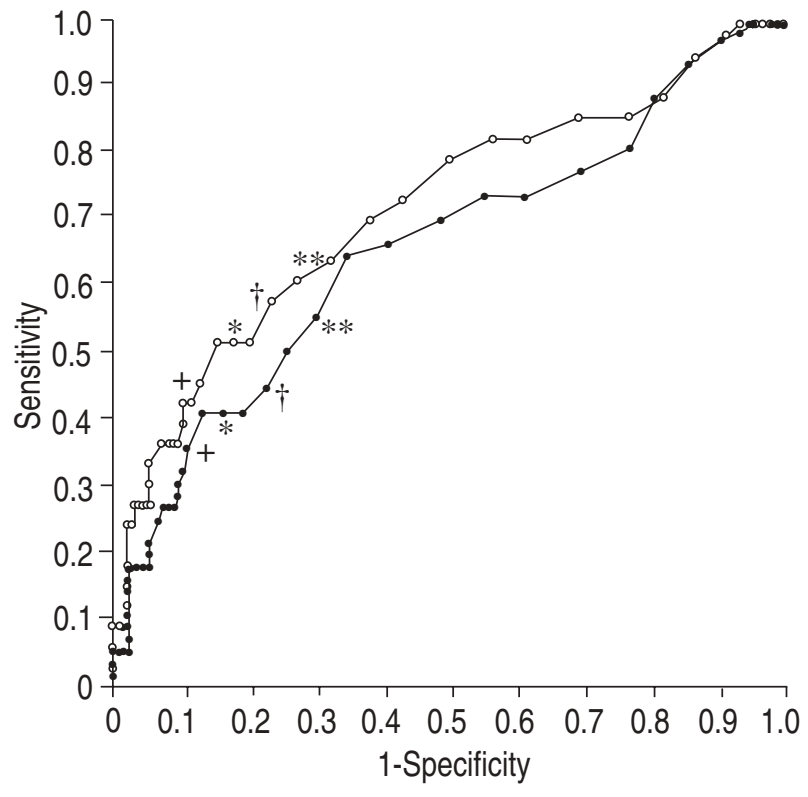

Fig. 2. - A comparison of the exercise challenge test with asthma history measured by parental questionnaire. - - - : child has current asthma (Q6 + Q2); ——: child has a history of asthma (Q6); for further definitions see legend to figure 1 .

Table 3. - The sensitivity and specificity of a positive exercise challenge for predicting responses to selected ISAAC questions

\begin{tabular}{|c|c|c|c|c|}
\hline \multicolumn{2}{|c|}{ Questions (response regarded as positive) } & \multirow{2}{*}{$\begin{array}{c}\text { Sensitivity } \\
0.40\end{array}$} & \multirow{2}{*}{$\begin{array}{c}\text { Specificity } \\
0.80\end{array}$} & \multirow{2}{*}{$\begin{array}{c}\begin{array}{c}\text { Youden's } \\
\text { Index }\end{array} \\
0.20\end{array}$} \\
\hline Q1. & $\begin{array}{l}\text { Has your child ever had wheezing or whistling } \\
\text { in the chest at any time in the past? (Yes) }\end{array}$ & & & \\
\hline Q2. & $\begin{array}{l}\text { Has your child had wheezing or whsitling in } \\
\text { the chest in the last } 12 \text { months? (Yes) }\end{array}$ & 0.54 & 0.78 & 0.31 \\
\hline Q4. & History of sleep disturbance due to wheeze. (Any) & 0.61 & 0.77 & 0.37 \\
\hline Q5. & History of speech limitation due to wheeze. (Yes) & 0.60 & 0.72 & 0.32 \\
\hline Q6. & Has your child ever had asthma? (Yes) & 0.45 & 0.78 & 0.22 \\
\hline Q7. & $\begin{array}{l}\text { In the last } 12 \text { months, has your child's chest } \\
\text { sounded wheezy during or after exercise? (Yes) }\end{array}$ & 0.60 & 0.77 & 0.37 \\
\hline Q8. & $\begin{array}{l}\text { In the last } 12 \text { months, has your child had a dry } \\
\text { cough at night, apart from a cough associated with } \\
\text { a cold or chest infection? (Yes) }\end{array}$ & 0.39 & 0.74 & 0.13 \\
\hline $\begin{array}{l}\text { Q2. \& } \\
\text { Q6. }\end{array}$ & History of recent wheeze and asthma (Yes) & 0.58 & 0.77 & 0.35 \\
\hline
\end{tabular}

For this table, a fall in FEV1 after exercise of greater than $12 \%$ is a positive test response. For complete wording of questions see table 1 .

(ISAAC Question 6) and current asthma (a positive response to ISAAC Questions 2 and 6). The cut-off points closest to the upper left hand corner of the graph for current asthma correspond to an FEV1 fall of 12 or $15 \%$. The exercise test generally had a higher sensitivity for current asthma than a history of asthma. The sensitivity and specificity of the exercise test using a cut-off of $12 \%$ fall in FEV1 is shown in table 3 in relation to individual ISAAC questions.

\section{Discussion}

This study was designed to assess the relationship between a physiological test of BHR and parental responses to an asthma questionnaire. The study involved a representative sample of school children in a geographical area. Selection bias was reduced by the high response rate (91\%), and special care was taken to ensure that the children of consenting families did the exercise challenge even if they were absent from school on the initial day of testing, because children with asthma or wheezing illness are likely to be over represented among school absentees [22].

The exercise challenge test used here was based on the protocol developed by HABY et al. [12], which overcomes the limitations of some earlier protocols that did not ensure mouth breathing or document exercise intensity and weather conditions. Forced expiratory volume was used as the measure of airflow because it has less variability than peak expiratory flow [15]. To reduce measurement error, 
the vitalograph was carefully calibrated. The protocol of the present study differed from that of HABY et al. [12] in that forced vital capacity (FVC) and peak expiratory flow (PEF) were not recorded, as these measures were not required for the exercise BHR analysis. Lung function measurements were conducted indoors because wind conditions made outdoor lung function tests difficult. In addition, indoor testing meant that the effect of cold air temperature causing initial bronchoconstriction before baseline readings was minimized. A limitation of the present study was that some children ran in atmospheric conditions when the absolute water content was above 10 $\mathrm{mgH}_{2} \mathrm{O} \cdot \mathrm{L}^{-1}$ because resources were inadequate to postpone exercise testing and reschedule it for more suitable conditions. An absolute water content above $10 \mathrm{mgH}_{2} \mathrm{O} \cdot \mathrm{L}^{-1}$ is thought to reduce the sensitivity of exercise challenge [23]. However, this would bias these results towards a more conservative estimate of sensitivity and, thus, the ROC curves may underestimate the agreement between exercise challenge response and parental questionnaire response.

From the exercise challenge data, the median percentage fall in FEV1 was significantly greater for children whose parents responded positively to questions on their child's history of wheeze, recent wheeze, nocturnal wheeze, exercise wheeze or asthma, used in the ISAAC epidemiological study $[1,8]$. The median percentage fall in FEV1 did not differ significantly by history of recent speech limitation due to wheeze, possibly because of the small number of positive responses. The exercise challenge results did not relate well to a history of a recent dry cough at night, other than from a cold or chest infection. Overall, the exercise challenge test results were in good agreement with the ISAAC questions, although this is, of necessity, a value judgement. The sensitivity and specificity estimates here are relatively high for a disease with complex features and temporal variability such as asthma, given that not all children with asthma may have exercise-induced bronchospasm on the particular day of testing. The specificity estimates for the test would be expected to rise if the exercise challenge was repeated over a 12 month period because the questions refer to period prevalence while the exercise challenge only measures point prevalence, an issue previously discussed by BURNEY et al. [24] in relation to bronchial response to histamine.

The Youden's Index for exercise challenge was higher for nocturnal wheeze, exercise wheeze and current asthma than a history of wheeze or whistling, asthma or recent dry nocturnal cough. The Youden's Index of a test is inversely related to the degree of bias in the test's estimate of the difference in disease prevalence between two communities [24]. This suggests that if exercise testing was conducted in different settings, it would provide a better indication of the difference in prevalence of exercise wheeze, nocturnal wheeze or current asthma than other clinical features of asthma measured by the ISAAC questionnaire, such as a history of wheeze or recent nocturnal cough. However, a larger study would be required to determine whether the differences between questions in relation to Youden's Index were important. The exercise test had the highest index for the question on nocturnal wheeze. A recent ISAAC validation study found that the written questions on nocturnal wheeze from the ISAAC protocol gave the highest calculated value for Youden's Index in relation to methacholine challenge [8].
Asthma has a clinical course of intermittent exacerbations with variable manifestations in different individuals. The prevalence of exercise-induced symptoms in patients with asthma has been reported to range from $40-90 \%$ in various studies, and part of this variation relates to differences in study methodology [9]. At present, asthma cannot be diagnosed using a "gold standard". BHR alone cannot be equated with clinical asthma [8]. Here, the use of questionnaire response as a measure of disease has limitations and should not be considered as the "gold standard" for test comparison. ROC curves are not used here to assess the performance of the exercise challenge in relation to disease. Rather, ROC curves are used to compare the agreement between the exercise challenge protocol and parental questionnaire response over a range of cut-off points for exercise BHR.

We believe ROC analysis should be more widely used in asthma epidemiology when assessing the performance of such markers for asthma, as it takes into account that bronchial responsiveness is a continuous variable. The choice of a critical level of bronchial reactivity to define asthma is a difficult one and the categorization of the continuous measure into a dichotomous variable may result in the loss of important information. Various cut-off points have been reported in relation to exercise challenge. In a recent review, a fall in FEV 1 of $15 \%$ or more was taken to indicate exercise-induced asthma [9]. НАBY et al. [12] used a fall in FEV1 of $13 \%$ or more as the criterion for BHR to exercise. This was calculated by determining the upper limit of normal (1.96 standard deviations above mean) for percentage fall in FEV1 in "normal" subjects (i.e. without diagnosed asthma, recent wheeze or atopy). KATTAN et al. [25] used a similar method (upper limit $=$ two SD above the mean of "normal" subjects), and thus classified a greater than $10 \%$ fall in FEV1 as abnormal. The protocol of a comparative survey of childhood asthma prevalence between New Zealand and South Wales, UK, specified a fall in PEF exceeding $15 \%$ as defining exercise-induced asthma [26]. It should be remembered that the cut-off values will also be a function of the method of estimation of percentage fall in FEV1. The optimal cut-off point (in terms of sensitivity and specificity) will be a clinical decision which will depend on issues such as the purpose of the test and whether the test is being performed in series or parallel with other tests for asthma [13]. For initial screening, a more sensitive test may be required, and thus a smaller fall in FEV1 may be suitable. However, a larger fall in FEV1 should be used as a cut-off point if the purpose of the test is to minimize the proportion of false positive tests.

In figures 1 and 2 , the shape of the ROC curves in the low range of 1 - Specificity (a rapid increase in sensitivity) is often found for tests which are able to discriminate diseased from nondiseased subjects. However, the further increase in sensitivity in the high range of 1 - Specificity is unusual. It partially reflects the fact that asthma is a disease with an intermittent course. Thus, one can expect that on a given day some of the children who have disease (that is, a history of exercise-related wheeze) may be "well" and thus test negative.

The written questionnaire upon which the international comparisons within ISAAC will be based has only recently been formally validated for the 13-14 year age group 
using methacholine challenge [8]. Here, the performance of respiratory exercise response in 7 year olds in relation to parental questionnaire responses over a range of cut-off values has been demonstrated using receiver operating characteristic curve analysis. This study provides new information for the 7 year age group, which should assist with the interpretation of the findings of ISAAC studies in relation to differences in asthma prevalence between and within communities.

Acknowledgements: The authors thank the Tasmanian Department of Education and Arts for their collaboration, participating schools and families, the Asthma Foundation of Tasmania for equipment loan, E. Gibson and K. Turner for data collection and T. Parkes for technical advice.

\section{References}

1. Asher MI, Keil U, Anderson HR, et al. International Study of Asthma and Allergies in Childhood (ISAAC): rationale and methods. Eur Respir J 1995; 8: 483-491.

2. Phelan PD. Asthma in children: epidemiology. Changing prevalence, patterns and treatment. Br Med J 1994; 308: 1584-1585.

3. Pearce N, Weiland S, Keil U, et al. Self-reported prevalence of asthma symptoms in children in Australia, England, Germany and New Zealand: an international comparison using the ISAAC protocol. Eur Respir $J$ 1993; 6: 1455-1461.

4. Burney PGJ, Laitinen LA, Perdrizet S, et al. Validity and repeatability of the IUATLD (1984) Bronchial Symptoms Questionnaire: an international comparison. Eur Respir J 1989; 2: 940-945.

5. Robertson CF, Heycock E, Bishop J, et al. Prevalence of asthma in Melbourne schoolchildren: changes over 26 yrs. Br Med J 1991; 302: 1116-1118.

6. Shaw RA, Crane J, O'Donnell TV, et al. The use of a videotaped questionnaire for studying asthma prevalence: a pilot study amongst New Zealand adolescents. Aust NZ J Med 1992; 157: 311-314.

7. Osterman JW, Armstrong BG, Ledoux E, et al. Comparison of French and English Versions of the American Thoracic Society respiratory questionnaire in a bilingual working population. Int J Epidemiol 1991; 20: 138-143.

8. Shaw R, Woodman K, Ayson M, et al. Measuring the prevalence of bronchial hyper responsiveness in children. Int J Epidemiol 1995; 24: 597-602.

9. McFadden ER Jr, Gilbert IA. Exercise-induced asthma. $N$ Engl J Med 1994; 330: 1362-1367.

10. Gilbert IA, Fouke JM, McFadden ER Jr. Intra-airway thermodynamics during exercise and hyperventilation in asthmatics. J Appl Physiol 1988; 64: 2167-2174.
11. Gilbert IA, Fouke JM, McFadden ER Jr. Heat and water flux in the intrathoracic airways and exercise-induced asthma. J Appl Physiol 1987; 63: 1681-1691.

12. Haby MM, Anderson SD, Peat JK, et al. An exercise challenge protocol for epidemiological studies of asthma in children: comparison with histamine challenge. Eur Respir J 1994; 7: 43-49.

13. Fletcher RH, Fletcher SW, Wagner EH. In: Fletcher RH, Fletcher SW, Wagner EH, Eds. Clinical epidemiology: the essentials 2nd edn. Baltimore, Williams and Wilkins, 1988; pp. 46-70.

14. Gibson HB, Silverstone H, Gandevia B, et al. Respiratory disorders of seven year old children in Tasmania: aims, methods and administration of the survey. Med J Aust 1969; 2: 201-205.

15. Strachan DP. Repeatability of ventilatory function measurements in a population survey of 7 year old children. Thorax 1989; 44: 474-479.

16. Miller MR, Pincock AC. Predicted values: how should we use them? Thorax 1988; 43: 265-267.

17. Silverman M, Anderson SD. Metabolic cost of treadmill exercise in children. J Appl Physiol 1972; 33: 696-698.

18. Morton AR, Fitch KD. Asthma. In: Skinner JS, ed. Exercise Testing and Exercise Prescription for Special Cases. Theoretical Basis and Clinical Application 2nd Edn. London, Lea \& Febiger, 1993: 211-228.

19. Sterk PJ. Airway responsiveness: standardised lung function testing. Official Statement of the European Respiratory Society. Eur Respir J 1993; 6 (Suppl. 16): 64-66.

20. Fisher LD, van Belle G. Biostatistics: A Methodology for the Health Sciences. New York, John Wiley \& Sons Inc., 1993

21. Youden WJ. Index for rating diagnostic tests. Cancer 1950; 3: 32-35.

22. Anderson HR, Bailey PA, Cooper JS, et al. Morbidity and school absence caused by asthma and wheezing illness. Arch Dis Child 1983; 58: 777-784.

23. Hahn A, Anderson SD, Morton AR, et al. A reinterpretation of the effect of temperature and water content of the inspired air in exercise-induced asthma. Am Rev Respir Dis 1984; 130: 575-579.

24. Burney PGJ, Chinn S, Britton JR, et al. What symptoms predict the bronchial response to histamine? Evaluation in a community survey of the bronchial symptoms questionnaire (1984) of The International Union Against Tuberculosis and Lung Disease. Int J Epidemiol 1989; 18: 165-173.

25. Kattan M, Keens TG, Mellis CM, et al. The response to exercise in normal and asthmatic children. J Pediatr 1978; 92: 718-721.

26. Barry DMJ, Burr ML, Limb ES. Prevalence of asthma among 12 year old children in New Zealand and South Wales: a comparative survey. Thorax 1991; 46: 405-409. 